\title{
Language neuroplasticity in brain tumour patients revealed by magnetoencephalography
}

Vitória Piai ${ }^{1,2}$, Elke De Witte ${ }^{3,4}$, Joanna Sierpowska ${ }^{1,2}$, Robert T. Knight ${ }^{6}$, Mitchel S. Berger ${ }^{4}$

1. Radboud University, Donders Institute for Brain Cognition and Behaviour, Donders Centre for Cognition, Nijmegen, the Netherlands

2. Radboud university medical center, Donders Institute for Brain Cognition and Behaviour, Department of Medical Psychology, Nijmegen, the Netherlands

3. Department of Clinical and Experimental Neurolinguistics, Center of Linguistics and Literary Studies, Free University of Brussels, Brussels, Belgium

4. Department of Neurological Surgery, University of California San Francisco, San Francisco, California

5.

6. University of California Berkeley, Department of Psychology, Helen Wills Neuroscience Institute, Berkeley, United States

Corresponding author:

Vitória Piai, $\mathrm{PhD}$

v.piai@donders.ru.nl

SOME AUTHOR NAMES ARE OMMITTED UNTIL THEIR FINAL APPROVAL HAS BEEN GIVEN 


\begin{abstract}
Language impairment in brain tumour patients may be missed since standardised tests fail to capture mild deficits. Neuroplasticity may also contribute to minimising language impairments. To address this possibility, we examined 14 patients with language dominant hemipsheric brain tumours prior to their first surgery using magnetoencephalography (MEG) imaging while they performed a demanding picture-word interference task. During pictureword interference, participants name pictures while ignoring distractor words. Brain tumour patients had the behavioural picture naming effects typically observed in healthy controls. By contrast, the MEG event-related effect had a right hemisphere source, in contrast to the classic left hemisphere source found in healthy individuals. This finding supports tumour induced neural reorganisation of language prior to surgery. We also identified one participant with a lesion affecting the left temporal lobe and underlying white-matter tracts who showed a deviant pattern in behaviour as well as in the MEG event-related responses. Our results provide support for neuroplasticity of language in brain tumours in the pre-surgical phase.
\end{abstract}

Keywords: aphasia; electrophysiology; neuroplasticity; N400; semantic processing 


\section{Introduction}

Brain tumour surgery aims to prolong survival by removing pathological tissue, while avoiding deficits (H. Duffau, 2007). This approach requires the use of tests that are sensitive enough to capture subtle impairment (Brownsett et al., 2019; De Witte et al., 2015; see also Rofes \& Miceli, 2014; Sierpowska et al., 2017). Protocols often make use of standard neuropsychological tests, which are sensitive to impairments in the moderate to severe range, such as those seen in stroke-induced aphasia. However, in the brain tumour population, subtle language and cognitive impairment may go unnoticed before surgery because standardised tests may fail to capture mild deficits (Satoer, Vincent, Smits, Dirven, \& Visch-brink, 2013). Additionally, brain plasticity may occur pre-surgically (Hugues Duffau, 2014), mitigating language impairment.

There is limited information on language impairment in brain tumour patients and on the relationship between impairment and lesion location (Satoer, Visch-Brink, Dirven, \& Vincent, 2016). The present study examines pre-surgical brain tumour patients performing an attentional demanding picture-naming task while their brain activity was monitored with magnetoencephalography (MEG). Speaking is an attentionally demanding task (Roelofs \& Piai, 2011) and the picture-word interference paradigm is sensitive to the attentional control demands necessary for naming a picture while ignoring distracting information (Piai \& Knight, 2018; Piai, Riès, \& Swick, 2016). In this paradigm, participants are asked to name a picture displayed on the screen while ignoring a distractor word, presented either auditorily or in written form superimposed onto the picture (see Figure 1 for an example). Previous studies have found that semantic interference (more difficult picture naming with categorically related distractors than with unrelated distractors, see Figure 1) implicates the left temporal lobe (Piai \& Knight, 2018; Piai, Roelofs, Jensen, Schoffelen, \& Bonnefond, 2014). By contrast, lexical interference (more difficult picture naming with lexical distractors than with a 
neutral XXX string, see Figure 1) implicates the ventrolateral prefrontal cortex (Piai et al., 2016). Brain tumours often grow along white-matter pathways in perisylvian language-related areas (Anderson, Damasio, \& Tranel, 1990), so the temporal lobe and the ventrolateral prefrontal cortex are cortical terminations whose functions may be affected due to the tumour. MEG provides a direct measure of neuronal activity in the subsecond time scale with enhanced localisation capacity. Certain neuronal "signatures" are well characterised in the literature in terms of their timing, associated brain areas, and sensitivity to experimental manipulations. These signatures may enable a better understanding of neuroplasticity as one can examine whether a certain signature typically found in a left-hemisphere brain area is now re-organised in a brain-lesioned individual (e.g., Piai, Meyer, Dronkers, \& Knight, 2017; Traut et al., 2018). There is a robust neurophysiological signature of lexical processing in picture naming, expressed as an amplitude modulation of the N400 event-related component (de Zubicaray \& Piai, 2019). The N400 is an event-related potential (ERP) that peaks approximately $400 \mathrm{~ms}$ post-stimulus onset and has multiple sources in the left temporal cortex (Lau, Phillips, \& Poeppel, 2008). In picture-word interference, enhanced N400 responses are found for unrelated relative to related picture-distractor pairs in the left temporal cortex (de Zubicaray \& Piai, 2019; Piai et al., 2014).

The present study assessed the feasibility of administering picture-word interference, an attentionally demanding task, in pre-surgical brain tumour patients while recording their brain activity at the sub-second timescale using MEG. We aimed to identify any deficits in word production in the pre-surgical phase, and examine patterns of neural reorganisation due to tumors. For that, we focused on the MEG counterpart of the N400 component, the N400m, as a functional measure of lexical processes. Given that this event-related response has a wellknown spatio-temporal characterisation, any changes in its timing or spatial components would support neuronal reorganisation of language functioning. 


\section{Methods}

\section{Participants}

Fourteen individuals ( 8 females; mean age at testing $=42.4$ ) with tumours in the languagedominant hemisphere undergoing pre-surgical MEG assessment participated in this study (for tumour sites, see Table 1). Twelve individuals were right handed and two were left handed, but all had tumours in the language-dominant hemisphere (thirteen in the left hemisphere, one in the right hemisphere), as defined by the laterality index measured with MEG during picture naming (Findlay et al., 2012) and confirmed by the Wada test (Wada, 1949) when necessary. All individuals were native speakers of English. The study was approved by the UCSF Institutional Review Board, and all participants gave written informed consent.

\section{Materials}

The experimental picture-word interference task was created using sixty coloured photographs chosen from the BOSS database (Brodeur, Dionne-Dostie, Montreuil, \& Lepage, 2010) or from the internet. The photographs belonged to ten different semantic categories, with six exemplars each (e.g., animals: cow, fish, horse, lion, own, rabbit). For each photograph, related distractor words were selected from names of the other categorycoordinate objects (e.g., pictured cow, distractor "fish"). Unrelated distractors were selected by recombining object names that were semantically and phonologically unrelated to the picture. Thus, all distractor words belonged to the response set. In the neutral condition, a series of five Xs appeared as a distractor. All participants saw each picture once in each condition. Pictures were presented on a white background on the centre of the screen. Distractors were presented in black font inside a white box, centred on the picture (see example in Figure 1). The picture-word trials were fully randomized, with one unique list per participant. Participants were instructed to name the picture and to ignore the distractor word. Both speed and accuracy were emphasized. 


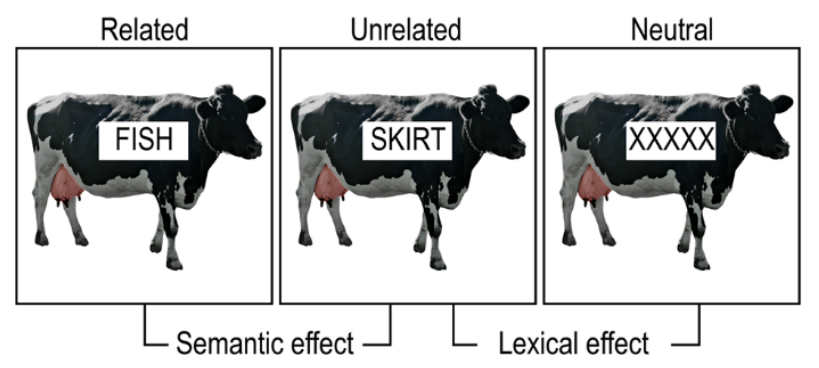

Figure 1. Example of picture-word interference stimuli for related (left), unrelated (middle), and neutral (right) distractors, and the corresponding interference effects.

\section{Procedure}

The presentation of stimuli and the recording of responses were controlled by E-prime 2.0 software (Psychology Software Tools, Pittsburgh, PA). Participants were laying down in supine position in an electrically and magnetically shielded room, with their heads in the opening of the MEG helmet. Stimuli were projected onto a screen placed above the participants. Vocal responses were recorded with a microphone along the MEG data. Trials began with a fixation cross presented on the centre of the screen for a variable duration, between 1.7 and $2.1 \mathrm{~s}$. Then, the picture-word stimulus was presented for $2 \mathrm{~s}$.

The MEG system (CTF VSM MedTech) contained 275 axial gradiometers. Three localisation coils were fixed to the nasion, left, and right pre-auricular points to monitor the position of participants' heads relative to the gradiometers. The data were low-pass filtered by an anti-aliasing filter (300 Hz cutoff), digitized at $1200 \mathrm{~Hz}$, and stored for offline analysis. A $3^{\text {rd }}$ order gradiometer configuration was used to reduce noise.

\section{Lesion analyses}

Lesions were drawn by a trained technician in the native space of participants' T1-weighted or T2-weighted magnetic resonance images (MRIs) and confirmed by a neurologist (RTK). The lesion delineations were subsequently normalised to the MNI template and checked again to confirm that no distortions occurred. Per cent damage to different areas was determined based on the Automated Anatomical Labeling template in MRIcroN (Rorden, Karnath, \& 
Bonilha, 2007). We also compared the precise lesion location of each individual with selected tractography reconstructions of white matter pathways obtained from a group of healthy controls (Rojkova et al., 2016). These analyses allowed us to quantify the proportion of overlap between the lesion's volume and the tract's volume using Tractotron software as part of the BCBtoolkit (Foulon et al., 2018, http://www.toolkit.bcblab.com). The selected pathways were chosen based on them passing through the MTG given the critical role of this area in language (Sierpowska et al., 2019; Turken \& Dronkers, 2011): the long and posterior segments of the arcuate fasciculus (AF), the inferior frontal occipital fasciculus (IFOF), and the inferior longitudinal fasciculus (ILF). Due to the heterogeneity in lesion distribution, we summarised the lesion profiles using hierarchical clustering over the proportion of damage to areas substantially impacted by the tumour, or areas previously associated with word production or picture-word interference, and for the four tracts that pass through the MTG, as mentioned above. The grey-matter areas selected for the analysis were: inferior temporal gyrus (ITG), middle temporal gyrus (MTG), including the pole, superior temporal gyrus (STG), including the pole, left inferior frontal gyrus (LIFG, part opercularis, pars orbicularis, and par triangularis), middle frontal gyrus (MFG), superior frontal gyrus (SFG), insula, and anterior cingular cortex (ACC). Clustering techniques group elements such that elements in one same cluster are more similar to each other than to elements in other clusters. Note that the values were selected for the participants' language-dominant hemisphere. The values used are shown in Table 2. The Euclidean distance was used, together with the Ward's criterion. Validation of the cluster solution was achieved via multiscale bootstrap resampling (1000 bootstraps, Suzuki \& Shimodaira, 2006). P values were derived from the Approximately Unbiased P value and we employed an alpha-level of .05. 


\section{Behavioural analyses}

Vocal responses were examined offline for dysfluencies or errors. The corresponding trials were excluded from all response time (RT) and MEG analyses. Naming RTs were calculated manually from the speech signal before trials were separated by condition. Single-trial data and analysis scripts are available via de Open Science Framework (http://tiny.cc/4q007y). Single-trial RT and accuracy were analysed with linear and logistic mixed-effects models, respectively Baayen, Davidson, \& Bates, 2008). Models were fitted with the lme4-package (version 3.4.4; Bates, Mächler, Bolker, \& Walker, 2015) in R (version 3.4.3, R Core Team, 2017). Both models included a fixed effect for distractor condition (related, unrelated, neutral; unrelated was the reference), and random slopes for the distractor condition by participant. Single-trial item information was not available. Significance of effects was obtained using the Satterthwaite approximation (lmerTest-package version 3.4.4, Kuznetsova, Brockhoff, \& Christensen, 2017). We also calculated standard (z) scores for each participant based on a jack-knifing approach (i.e., the semantic and lexical effects for a given individual are compared to the group without that individual).

\section{MEG analyses}

For the MEG data, analyses were performed using FieldTrip (version 20171231, Oostenveld, Fries, Maris, \& Schoffelen, 2011) in MatlabR2017b. The data were detrended, down-sampled offline to $600 \mathrm{~Hz}$, and segmented into epochs from $.3 \mathrm{~s}$ pre-stimulus to $1 \mathrm{~s}$ post-stimulus. MEG epochs were inspected and excessively noisy channels were removed. Independent component analysis was then used to correct for artefacts, including eye movements (Jung et al., 2000). Artefact- and error-free data comprised on average 56, 55, and 57 trials for the related, unrelated, and neutral conditions, respectively. The signal in single trials was lowpass filtered with a zero-phase shift Butterworth filter with a cut-off frequency of $30 \mathrm{~Hz}$. Event-related fields were then computed by averaging the trials for each condition and 
participant separately, followed by baseline correction using the averaged activity in the interval of $-300 \mathrm{~ms}$ to $0 \mathrm{~ms}$ relative to picture onset.

Following the evidence that semantic interference (i.e., related versus unrelated conditions) is mainly associated with electrophysiological differences in the N400 time window and implicates mainly the left temporal lobe (de Zubicaray \& Piai, 2019), the activity for the related and unrelated conditions was averaged around the N400 time window (i.e., $350-450 \mathrm{~ms}$ ) over the left posterior sensors available for all participants. This "N400 activity" was used descriptively to examine the pattern of brain responses over the whole group. For the lexical effect, no information in the literature was available to motivate a specific spatiotemporal dimension of the data. Therefore, this analysis was not conducted for the lexical effect.

In addition, for inferential statistics of the event-related fields, we ran non-parametric cluster-based permutation tests for both semantic and lexical effects (Maris \& Oostenveld, 2007) with no a-priori information on sensors or time points (the window of picture onset to $600 \mathrm{~ms}$ post onset was examined). Non-parametric cluster-based permutation effectively controls the false alarm rate at the nominal level of .05 , while comparing the sensors and time points between conditions. The largest cluster in size of adjacent sensors and time points exhibiting a similar difference between the conditions assessed was identified by means of dependent-samples t-tests thresholded at an alpha level of .05. The permutation $p$ value was calculated using the Monte Carlo method with 1,000 random permutations. A Monte Carlo cluster $p$ value below 5\% (two-tailed testing) was considered significant.

\section{Results}

\section{Lesion profile}

Figure 2 shows how participants are grouped in clusters as a function of their grey- (left) and white-matter (right) lesion profiles. The y-axis indicates how dissimilar, according to the 
Euclidean distance, the individual data points and clusters are from each other. Significant clusters are indicated by the grey outlines. For the grey matter, three different clusters were identified. The lesion overlap of participants pertaining to these three different clusters is shown in Figure 3 (left). Participants 1 and 3 formed one cluster, characterised by lesions overlapping in the ITG. Participants 2 and 12 formed another cluster, characterised by lesions overlapping in the insula. Participants 5, 6, 7, 8, 9, 10, 11 formed the third cluster, with inconsistent lesion overlap. Participants 4, 13, and 14 did not enter any clusters, indicating that these three participants have more particular lesions, and are shown separately in Figure 3 (right). For the white matter, one large cluster was identified, including all but participant 13. Thus, the lesion profile analysis indicates that participant 13 had a lesion that did not cluster with other participants' lesions both at the grey- and white-matter levels.
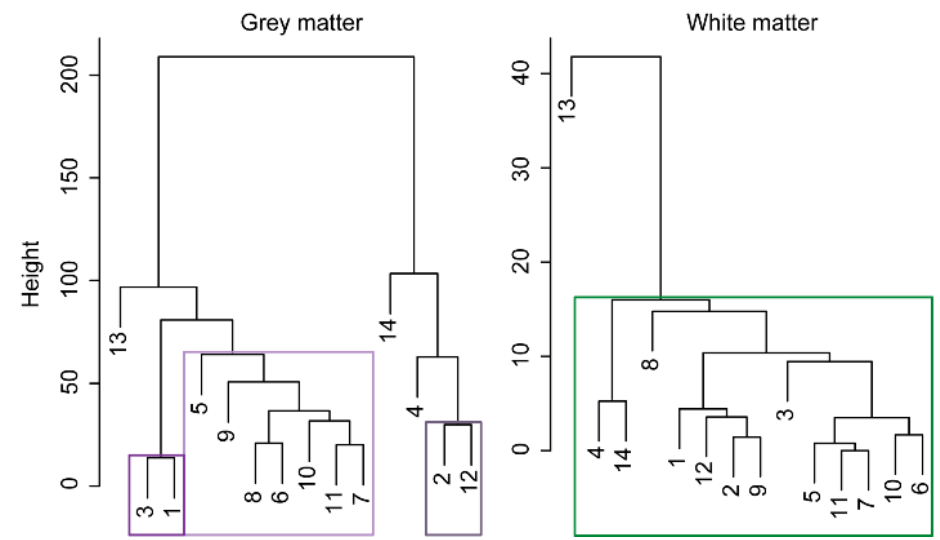

Figure 2. Dendrograms of the lesion clusters. Significant clusters are indicated by the coloured outlines. Left. Lesion in grey matter (proportion damage): anterior cingulate, insula, inferior frontal gyrus: pars opercularis, orbitalis, and triangularis, middle frontal gyrus, superior frontal gyrus, inferior temporal gyrus, middle temporal gyrus and pole, and superior temporal gyrus and pole. Right. Lesion in white matter (proportion damage): arcuate fasciculus-long segment, arcuate fasciculus-posterior segment, inferior frontal occipital fasciculus, inferior longitudinal fasciculus.

\section{Behavioural results}

Overall error rates are presented in Table 1 for each participant individually. At the group level, no difference was found in accuracy between the related and unrelated conditions $(4.9 \%$ 
vs $5.2 \%$, respectively, $\mathrm{b}$ estimate $=-.120$, S.E. $=.243, \mathrm{z}=.494, \mathrm{p}=.622$ ). More errors were made in the unrelated than in the neutral condition, that is, the lexical interference effect $(5.2 \%$ vs $2.4 \%$, respectively, b estimate $=-.823$, S.E. $=.273, \mathrm{z}=-3.019, \mathrm{p}=.003)$.
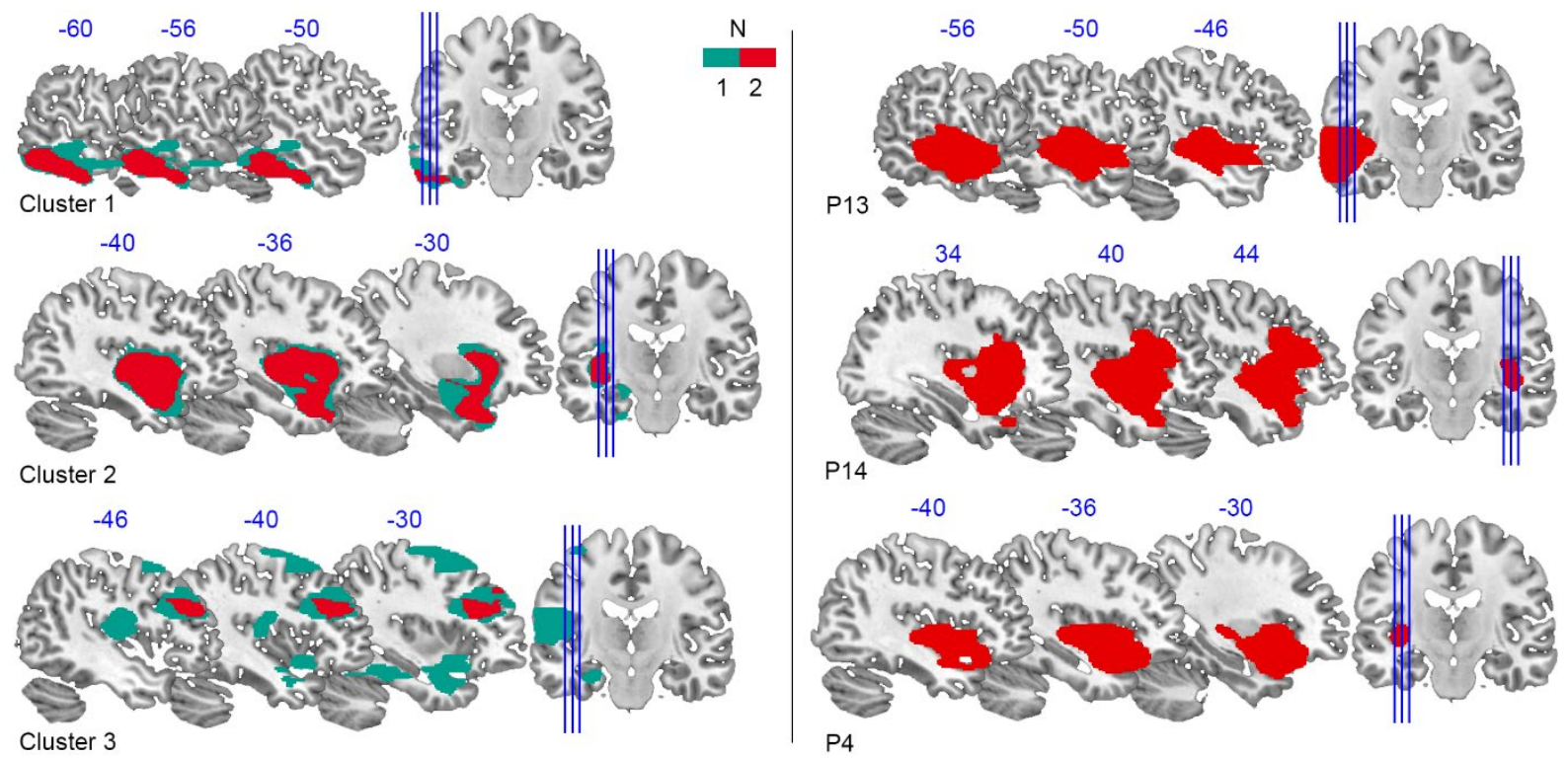

Figure 3. Lesion overlap. Left. Lesion overlap of the participants for the three identified clusters. The colour scale indicates the number of participants for which the overlap consists of. For clusters 1 and 2, with two participants each, $\mathrm{N}=1$ (green) corresponds to $50 \%$ overlap and $\mathrm{N}=2$ (red) corresponds to $100 \%$ overlap. For cluster 3, based on seven participants, $\mathrm{N}=$ 1 (green) corresponds to $14 \%$ overlap and $\mathrm{N}=2$ (red) corresponds to $29 \%$ overlap. Right. Lesion delineation for individual participants not pertaining to any cluster.

Figure 4 (left panel) shows the RTs for each participant and condition. The median RTs were $1.03 \mathrm{~s}$ for the related condition, $.978 \mathrm{~s}$ for the unrelated, and $.913 \mathrm{~s}$ for the neutral. Both lexical and semantic interference effects were found (semantic: $b$ estimate $=.06$, S.E. $=$ $.01, \mathrm{t}=4.66, \mathrm{p}<.001$; lexical: $\mathrm{b}$ estimate $=-.06$, S.E. $=.02, \mathrm{t}=-2.73, \mathrm{p}=.017)$. For the semantic effect, descriptively all participants show semantic interference. For the lexical effect, descriptively three participants showed facilitation (Participants 8, 11, and 12). Figure 4 (middle and right) shows the standard score for each participant for both the semantic (middle) and lexical (right) effects. Participants 13 and 14 showed semantic interference effects 1.5 standard deviations larger than the group mean. For the lexical effect, Participants 
6 and 13 showed lexical interference effects 1.5 standard deviations larger than the group mean, whereas Participant 12 showed a lexical facilitation effect 1.5 standard deviations away from the group mean. Participant 13 is the only individual to show deviant effects for both semantic and lexical interference following our jack-knifing approach. We note that this individual did not have overall language production problems, as shown for example by his high accuracy in picture naming (Table 1). We further tested the abnormality in the scores of Participant 13 for the semantic and lexical effects using a modified paired-samples test appropriate for single cases (Crawford, Howell, \& Garthwaite, 1998). For semantic interference, the effect for Participant 13 was discrepant with the control sample, $t=-5.044$, (estimated percentage of normal population more extreme than Participant $13=0.014 \%$ ). For lexical interference, the effect for Participant 13 was also discrepant with the control sample, but less so than the semantic interference effect, $t=-1.754$ (estimated percentage of normal population more extreme than Participant $13=5.248 \%$ ).
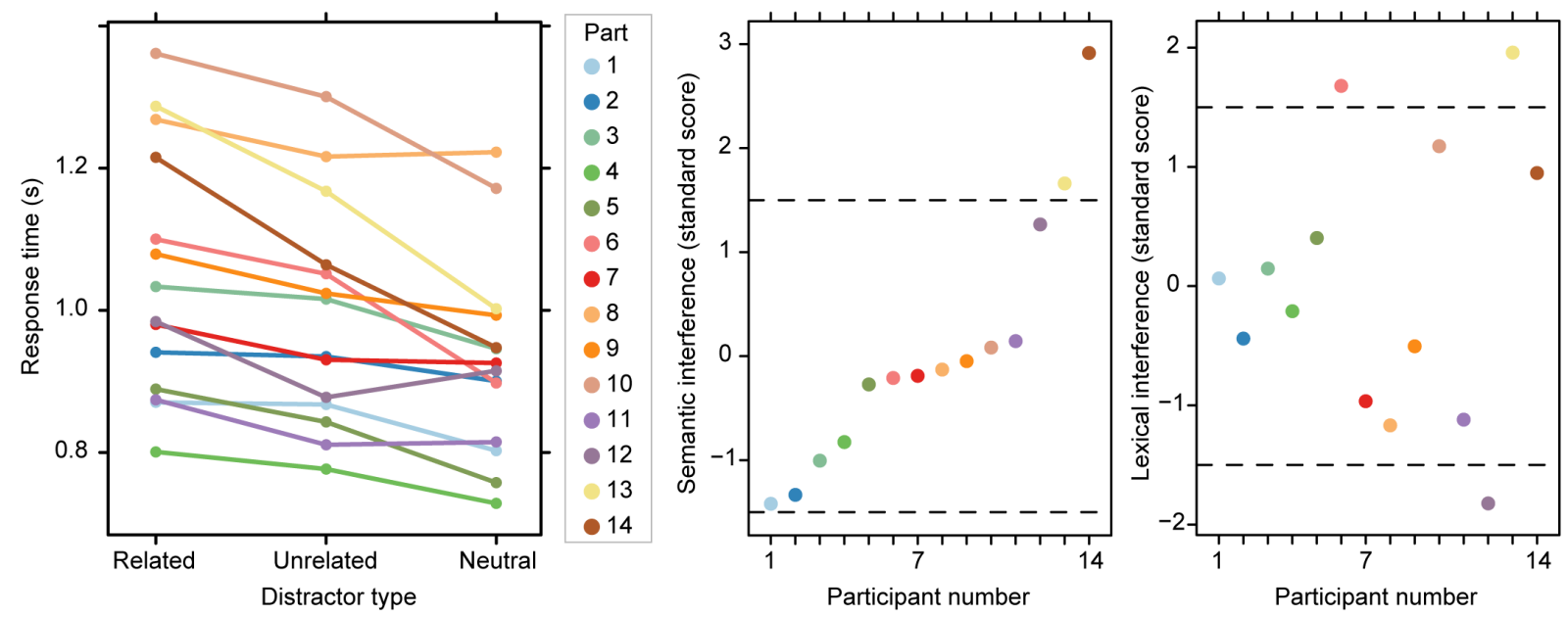

Figure 4. Behavioural results. Each colour indicates one participant (Part = participant). Left. Median response time per participant for each distractor type. Right. Standardised semantic (left) and lexical (right) interference effects per participant. Each dot represents one participant. Dashed horizontal lines indicate \pm 1.5 standard deviation. 


\section{MEG results}

Figure 5A shows the averaged N400 activity between $350-450$ ms over left posterior sensors for each participant for the related and unrelated conditions (left panel) and the semantic effect (related minus unrelated, right panel). Participant 13 presents a deviant pattern over left posterior sensors in the ordering of the conditions compared to the rest of the group, $t$ $=-2.714$, estimated percentage of normal population more extreme than Participant $13=$ $0.941 \%$ (Figure 5A, right).
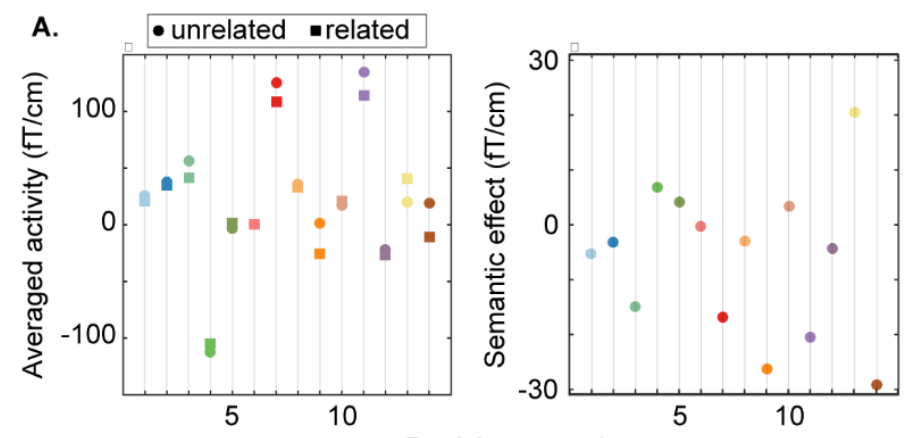

B.
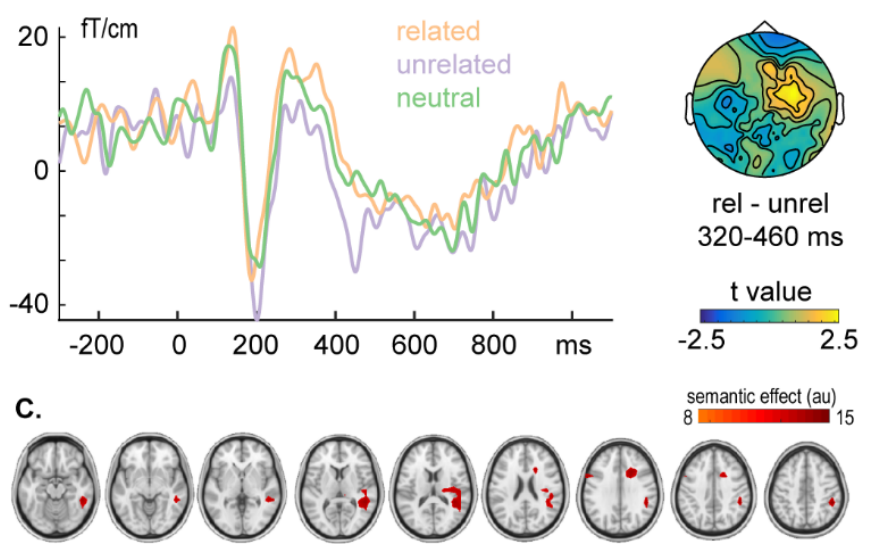

Figure 5.A. Averaged activity between $350-450 \mathrm{~ms}$ over left posterior sensors for each participant for the unrelated (circle) and related (square) conditions (left panel) and the semantic effect (related minus unrelated, right panel). Each dot is one participant. B. Eventrelated fields for the related (rel), unrelated (unrel), and neutral conditions for the entire sample averaged over the sensors showing the most pronounced differences for the semantic effect, which can be seen on the right. C. Source localisation on the group level of the semantic effect in the significant time window.

Regarding the inferential analyses of the MEG event-related responses, no significant clusters were identified for the lexical effect. By contrast, one significant cluster was identified for the semantic effect (Monte Carlo $p=.010$, two-tailed). Figure 5B shows the 
ERFs of the entire sample for the three conditions, averaged over the sensors showing the most pronounced differences for the semantic effect. The difference between the related and unrelated conditions was most pronounced in the time window between 320 to $460 \mathrm{~ms}$ over right-hemisphere sensors. We employed a linearly constrained minimum variance beamforming approach in the time domain (Van Veen, van Drongelen, Yuchtman, \& Suzuki, 1997) to localise the sources of the effect in this time range. The source localisation results are shown in Figure 5C. The source localisation indicates that the modulation is signal amplitude in the N400 time window originates in the right hemisphere, most prominently in the temporal lobe, but also inferior parietal lobe and superior frontal gyrus. Given that Patient 14 had a brain tumour in the right, language-dominant hemisphere, we repeated the analyses of the ERFs without Patient 14. The results of this additional analysis are shown in the Supplement. The pattern of N400 effect with right-lateralised topography in the sample of 13 patients with tumours in the left language-dominant hemisphere was virtually identical to the pattern shown in Figure 5B. This finding underscores that the right-hemisphere shift observed in the whole group is not driven by the individual with right-hemisphere language dominance.

\section{Discussion}

We assessed the feasibility of administering picture-word interference during MEG recordings in pre-surgical brain tumour patients and examined tumour-induced neuronal reorganisation. On the group level, we observed the expected lexical interference and semantic interference effects in the picture naming times and the N400-like event-related responses associated with the semantic effect. The N400-semantic effect had sources in the right temporal cortex in tumour patients in contrast to the left hemisphere N400 effect typically found in healthy controls (de Zubicaray \& Piai, 2019; Piai et al., 2014). One participant with a lesion affecting the left temporal lobe and underlying white-matter tracts 
showed a deviant pattern in behaviour as well as in N400 event-related responses. We discuss each of these effects below.

We observed the expected lexical interference and semantic interference effects in the picture naming times, and in the error rates for the lexical interference effect. This is in line with previous research (Damian \& Bowers, 2003; Glaser \& Düngelhoff, 1984; La Heij, 1988; Piai et al., 2016; Roelofs, 2003), including the finding that a semantic interference effect in the error rates is not typically observed (e.g., Piai \& Knight, 2018). By using a jack-knifing approach, we identified two participants performing more poorly than 1.5 standard deviations from the group's mean with respect to the semantic interference effect and to the lexical interference effect. One participant (Participant 13) showed poorer performance for both effects. The analyses on the profile of the lesions identified different clusters at the grey- and white-matter levels. At the grey-matter level, three participants showed a more distinct lesion profile, not entering any clusters (including Participant 13). By contrast, at the white-matter level, all participants, with the exception of Participant 13, were clustered together, indicating that Participant 13 had a unique white-matter lesion pattern. The planned MEG analyses for the semantic effect (de Zubicaray \& Piai, 2019) also identified a pattern of activity over left posterior sensors that was different for Participant 13. The discrepant patterns observed for this participant were confirmed by statistical analyses appropriate for single-subject comparisons to a control group (Crawford et al., 1998).

We were unable to identify specific disease characteristics that could explain the observed pattern. Participant 13 had a fast-growing type of tumour, grade III anaplastic astrocytoma. Whereas Participants 3 and 4 also had high-grade tumours, only in Participant 13 did the tumour infiltrate large parts of the language-dominant temporal lobe. In particular, this was the only individual with such a large portion of MTG involvement, and damage in all ventral and dorsal tracts inspected. The combination of a fast-growing tumour, which limits 
the time for functional reorganisation (Desmurget, Bonnetblanc, \& Duffau, 2007; Kong, Gibb, \& Tate, 2016), in this critical location, i.e., the language-dominant MTG and the fibres passing through it (Dronkers, Wilkins, Van Valin, Redfern, \& Jaeger, 2004; Griffis, Nenert, Allendorfer, \& Szaflarski, 2017; Piai \& Knight, 2018; Schwartz et al., 2009; Turken \& Dronkers, 2011), could potentially explain the deficits observed across both lexical and semantic effects.

Regarding the MEG analyses, for the semantic effect, a difference was found at the group level between the related and unrelated conditions in the expected time window (de Zubicaray \& Piai, 2019), but with an altered topographical distribution. The effect was shifted to the right and confirmed by source localisation analyses, suggesting involvement of the right hemisphere at the group level. Evidence has accumulated for the right hemisphere's role in language functioning following damage to the language-dominant left hemisphere due to brain tumours (De Witte et al., 2014; Thiel et al., 2005; see also Traut et al., 2019, for right hemisphere shifts in the post-operative phase) similarly as found for stroke-induced lesions using MEG (e.g., Kielar, Deschamps, Jokel, \& Meltzer, 2016; Piai et al., 2017). This phenomenon could explain the present findings. Interestingly, the pattern found in the right hemisphere resembles the N400 response usually found over the left hemisphere both in timing and amplitude modulation as a function of the distractor word (de Zubicaray \& Piai, 2019; Piai et al., 2014). This finding highlights the advantage of using electrophysiological techniques to understand lesion-dependent language deficits and plasticity. Language-related neural signatures can provide temporal and spatial information in addition to magnitude of effects in response to experimental manipulations. This enables examining whether these signatures have shifted in location due to a lesion, providing important information for understanding neuroplasticity. This shift may also explain why most individuals do not present with language deficits despite the brain lesions in the dominant language hemisphere. 
One limitation of this study is that the distribution of the lesions in the present sample was heterogeneous, but this heterogeneity was in fact helpful for identifying a possible lesionsymptom relationship. Another limitation of the present study is that the number of trials was not sufficient for analysing the MEG responses as a function of experimental condition at the within-participant level.

\section{Conclusions}

The replication of the classic lexical and semantic interference effects behaviourally and the MEG semantic effect attest to the reliability and validity of the approach. The present results provide support for neuroplasticity in the pre-surgical phase, with the right hemisphere performing similar neuronal computations (reflected in MEG N400 event-related responses) as the left hemisphere typically performs (see also Piai, Meyer, Dronkers, \& Knight, 2017). Additionally, we identified word-production deficits in one participant with a unique lesion profile, also affecting the $\mathrm{N} 400 \mathrm{~m}$ pattern of results. An important question for future research is whether the behavioural and/or electrophysiological patterns observed pre-surgically with such a challenging word-production task is predictive of an individual's deficits intraoperatively and recovery post-operatively. 


\section{References}

Anderson, S. W., Damasio, H., \& Tranel, D. (1990). Neuropsychological impairments associated with lesions caused by tumor or stroke. Archives of Neurology, 47(4), 397405.

Baayen, R. H., Davidson, D. J., \& Bates, D. M. (2008). Mixed-effects modeling with crossed random effects for subjects and items. Journal of Memory and Language, 59(4), 390412. https://doi.org/10.1016/j.jml.2007.12.005

Bates, D., Mächler, M., Bolker, B., \& Walker, S. (2015). Fitting Linear Mixed-Effects Models Using lme4. Journal of Statistical Software, 67(1). https://doi.org/10.18637/jss.v067.i01

Brodeur, M. B., Dionne-Dostie, E., Montreuil, T., \& Lepage, M. (2010). The Bank of Standardized Stimuli (BOSS), a new set of 480 normative photos of objects to be used as visual stimuli in cognitive research. PloS One, 5(5), e10773. https://doi.org/10.1371/journal.pone.0010773

Brownsett, S. L. ., Ramajoo, K., Copland, D., McMahon, K. ., Robinson, G., Drummond, K., ... De Zubicaray, G. (2019). Language deficits following dominant hemisphere tumour resection are significantly underestimated by syndrome-based aphasia assessments. Aphasiology. https://doi.org/10.1080/02687038.2019.1614760

Crawford, J. R., Howell, D. C., \& Garthwaite, P. H. (1998). Payne and Jones revisited: estimating the abnormality of test score differences using a modified paired samples $\mathrm{t}$ test. Journal of Clinical and Experimental Neuropsychology, 20(6), 898-905. https://doi.org/10.1076/jcen.20.6.898.1112

Damian, M. F., \& Bowers, J. S. (2003). Locus of semantic interference in picture-word interference tasks. Psychonomic Bulletin \& Review, 10(1), 111-117. Retrieved from http://www.ncbi.nlm.nih.gov/pubmed/12747497

De Witte, E., Satoer, D., Robert, E., Colle, H., Verheyen, S., Visch-Brink, E., \& Mari??n, P. (2015). The Dutch Linguistic Intraoperative Protocol: A valid linguistic approach to awake brain surgery. Brain and Language, 140, 35-48. https://doi.org/10.1016/j.bandl.2014.10.011

De Witte, E., Van Hecke, W., Dua, G., De Surgeloose, D., Moens, M., \& Mariën, P. (2014). Atypical cerebral language dominance in a right-handed patient: An anatomoclinical study. Clinical Neurology and Neurosurgery, 117(January), 12-21. https://doi.org/10.1016/j.clineuro.2013.11.014

de Zubicaray, G. I., \& Piai, V. (2019). Investigating the spatial and temporal components of speechproduction. In G. I. de Zubicaray \& N. O. Schiller (Eds.), The Oxford Handbook of Neurolinguistics. Oxford University Press.

Desmurget, M., Bonnetblanc, F., \& Duffau, H. (2007). Contrasting acute and slow-growing lesions: A new door to brain plasticity. Brain, 130(4), 898-914.

https://doi.org/10.1093/brain/awl300

Dronkers, N. F., Wilkins, D. P., Van Valin, R. D., Redfern, B. B., \& Jaeger, J. J. (2004). Lesion analysis of the brain areas involved in language comprehension. Cognition, 92(12), 145-177. https://doi.org/10.1016/j.cognition.2003.11.002

Duffau, H. (2007). Contribution of cortical and subcortical electrostimulation in brain glioma surgery: Methodological and functional considerations. Neurophysiologie 
Clinique/Clinical Neurophysiology, 37(6), 373-382.

https://doi.org/10.1016/j.neucli.2007.09.003

Duffau, H. (2014). The huge plastic potential of adult brain and the role of connectomics: New insights provided by serial mappings in glioma surgery. Cortex, 58, 325-337. https://doi.org/10.1016/j.cortex.2013.08.005

Findlay, A. M., Ambrose, J. B., Cahn-Weiner, D. a., Houde, J. F., Honma, S., Hinkley, L. B. N., ... Kirsch, H. E. (2012). Dynamics of hemispheric dominance for language assessed by magnetoencephalographic imaging. Annals of Neurology, 71(5), 668-686. https://doi.org/10.1002/ana.23530

Foulon, C., Cerliani, L., Kinkingnéhun, S., Levy, R., Rosso, C., Urbanski, M., ... Thiebaut de Schotten, M. (2018). Advanced lesion symptom mapping analyses and implementation as BCBtoolkit. GigaScience, 7(3). https://doi.org/10.1093/gigascience/giy004

Glaser, W. R., \& Düngelhoff, F. J. (1984). The time course of picture-word interference. Journal of Experimental Psychology: Human Perception and Performance, 10(5), 640654. Retrieved from http://www.ncbi.nlm.nih.gov/pubmed/6238124

Griffis, J. C., Nenert, R., Allendorfer, J. B., \& Szaflarski, J. P. (2017). Damage to white matter bottlenecks contributes to language impairments after left hemispheric stroke. NeuroImage: Clinical, 14, 552-565. https://doi.org/10.1016/j.nicl.2017.02.019

Jung, T. P., Makeig, S., Humphries, C., Lee, T. W., M.J., M., Iragui, V., \& Sejnowski, T. J. (2000). Removing electroencephalographic artifacts by blind source separation. Psychophysiology, 37, 163-178.

Kielar, A., Deschamps, T., Jokel, R., \& Meltzer, J. A. (2016). Functional reorganization of language networks for semantics and syntax in chronic stroke: Evidence from MEG. Human Brain Mapping, 37(8), 2869-2893. https://doi.org/10.1002/hbm.23212

Kong, N. W., Gibb, W. R., \& Tate, M. C. (2016). Neuroplasticity: insights from patients harboring gliomas. Neural Plasticity, 2016, 1-12. https://doi.org/10.1155/2016/2365063

Kuznetsova, A., Brockhoff, P. B., \& Christensen, R. H. B. (2017). lmerTest Package: Tests in Linear Mixed Effects Models. Journal of Statistical Software, 82(13). https://doi.org/10.18637/jss.v082.i13

La Heij, W. (1988). Components of Stroop-like interference in picture naming. Memory and Cognition, 16(5), 400-410. Retrieved from http://www.ncbi.nlm.nih.gov/pubmed/3173088

Lau, E. F., Phillips, C., \& Poeppel, D. (2008). A cortical network for semantics: (de)constructing the N400. Nature Reviews Neuroscience, 9, 920-933. https://doi.org/10.1038/nrn2532

Maris, E., \& Oostenveld, R. (2007). Nonparametric statistical testing of EEG- and MEG-data. Journal of Neuroscience Methods, 164, 177-190. https://doi.org/10.1016/j.jneumeth.2007.03.024

Oostenveld, R., Fries, P., Maris, E., \& Schoffelen, J.-M. (2011). FieldTrip: Open source software for advanced analysis of MEG, EEG, and invasive electrophysiological data. Computational Intelligence and Neuroscience, 2011, 1-9. https://doi.org/10.1155/2011/156869 
Piai, V., \& Knight, R. T. (2018). Lexical selection with competing distractors: Evidence from left temporal lobe lesions. Psychonomic Bulletin \& Review, 25(2), 710-717. https://doi.org/10.3758/s13423-017-1301-0

Piai, V., Meyer, L., Dronkers, N. F., \& Knight, R. T. (2017). Neuroplasticity of language in left-hemisphere stroke: Evidence linking subsecond electrophysiology and structural connections. Human Brain Mapping, 38(6), 3151-3162. https://doi.org/10.1002/hbm.23581

Piai, V., Riès, S. K., \& Swick, D. (2016). Lesions to Lateral Prefrontal Cortex Impair Lexical Interference Control in Word Production. Frontiers in Human Neuroscience, 9(January), 1-13. https://doi.org/10.3389/fnhum.2015.00721

Piai, V., Roelofs, A., Jensen, O., Schoffelen, J.-M., \& Bonnefond, M. (2014). Distinct patterns of brain activity characterise lexical activation and competition in spoken word production. PloS One, 9(2), e88674. https://doi.org/10.1371/journal.pone.0088674

Roelofs, A. (2003). Goal-referenced selection of verbal action: Modeling attentional control in the Stroop task. Psychological Review, 110(1), 88-125. https://doi.org/10.1037/0033295X.110.1.88

Roelofs, A., \& Piai, V. (2011). Attention demands of spoken word planning: a review. Frontiers in Psychology, 2(307), 1-14. https://doi.org/10.3389/fpsyg.2011.00307

Rofes, A., \& Miceli, G. (2014). Language mapping with verbs and sentences in awake surgery: a review. Neuropsychology Review, 24(2), 185-199. https://doi.org/10.1007/s11065-014-9258-5

Rojkova, K., Volle, E., Urbanski, M., Humbert, F., Dell'Acqua, F., \& Thiebaut de Schotten, M. (2016). Atlasing the frontal lobe connections and their variability due to age and education: a spherical deconvolution tractography study. Brain Structure and Function, 221(3), 1751-1766. https://doi.org/10.1007/s00429-015-1001-3

Rorden, C., Karnath, H.-O., \& Bonilha, L. (2007). Improving lesion-symptom mapping. Journal of Cognitive Neuroscience, 19, 1081-1088.

Satoer, D., Vincent, A., Smits, M., Dirven, C., \& Visch-brink, E. (2013). Spontaneous speech of patients with gliomas in eloquent areas before and early after surgery. Acta Neurochirurgica, 155, 685-692. https://doi.org/10.1007/s00701-013-1638-8

Satoer, D., Visch-Brink, E., Dirven, C., \& Vincent, A. (2016). Glioma surgery in eloquent areas: can we preserve cognition? Acta Neurochirurgica, 158(1), 35-50. https://doi.org/10.1007/s00701-015-2601-7

Schwartz, M. F., Kimberg, D. Y., Walker, G. M., Faseyitan, O., Brecher, A., Dell, G. S., \& Coslett, H. B. (2009). Anterior temporal involvement in semantic word retrieval: voxelbased lesion-symptom mapping evidence from aphasia. Brain, 132, 3411-3427. https://doi.org/10.1093/brain/awp284

Sierpowska, J., Gabarrós, A., Fernandez-Coello, A., Camins, À., Castañer, S., Juncadella, M., ... Rodríguez-Fornells, A. (2017). Words are not enough: nonword repetition as an indicator of arcuate fasciculus integrity during brain tumor resection. Journal of Neurosurgery, 126(2), 435-445. https://doi.org/10.3171/2016.2.JNS151592

Sierpowska, J., Gabarrós, A., Fernández-Coello, A., Camins, À., Castañer, S., Juncadella, M., ... Rodríguez-Fornells, A. (2019). White-matter pathways and semantic processing: 
intrasurgical and lesion-symptom mapping evidence. NeuroImage: Clinical, 22(May 2018), 101704. https://doi.org/10.1016/j.nicl.2019.101704

Suzuki, R., \& Shimodaira, H. (2006). Pvclust: an R package for assessing the uncertainty in hierarchical clustering. Bioinformatics, 22, 1540-1542.

Team, R. C. (2017). R: A language and environment for statistical computing. R Foundation for Statistical Computing. Vienna, Austria. Retrieved from https://www.r-project.org

Thiel, A., Habedank, B., Winhuisen, L., Herholz, K., Kessler, J., Haupt, W. F., \& Heiss, W. D. (2005). Essential language function of the right hemisphere in brain tumor patients. Annals of Neurology, 57(1), 128-131. https://doi.org/10.1002/ana.20342

Traut, T., Sardesh, N., Bulubas, L., Findlay, A., Honma, S. M., Mizuiri, D., ... Tarapore, P. E. (2019). MEG imaging of recurrent gliomas reveals functional plasticity of hemispheric language specialization. Human Brain Mapping, 40(4), 1082-1092. https://doi.org/10.1002/hbm.24430

Turken, A. U., \& Dronkers, N. F. (2011). The neural architecture of the language comprehension network: converging evidence from lesion and connectivity analyses. Frontiers in Systems Neuroscience, 5(February), 1. https://doi.org/10.3389/fnsys.2011.00001

Van Veen, B. D., van Drongelen, W., Yuchtman, M., \& Suzuki, a. (1997). Localization of brain electrical activity via linearly constrained minimum variance spatial filtering. IEEE Transactions on Bio-Medical Engineering, 44(9), 867-880. https://doi.org/10.1109/10.623056

Wada, J. (1949). A new method for determination of the side of cerebral speech dominance: a preliminary report on the intracarotid injection of sodium amytal in man. Iqakaa Te Seibutzuqaki, 14, 221-222. 
Table 1. Characteristics of the participants.

Participant Pathology

1 Astrocytoma

2 F 34

6.1

2 Diffuse astrocytoma

2 F 31

5.6

3 Glioblastoma

$\begin{array}{lll}4 & \text { M } & 77\end{array}$

3.9

4 Anaplastic astrocytoma

$3 \quad$ M 27

5 NA

NA

F $\quad 36$

6 Oligodendroglioma

$2 \mathrm{~F} \quad 35$

1.7

7 Oligodendroglioma

2 M 41

1.7

8 Diffuse astrocytoma

$2 \mathrm{M} \quad 51$

10

9 Oligodendroglioma

$2 \mathrm{M} \quad 58$

3. 9

10 Oligodendroglioma

$\begin{array}{lll}2 & \mathrm{~F} & 47\end{array}$

11 Meningioangiomatosis

NA**

F 22

2.8

12 Oligoastrocytoma

$\begin{array}{lll}2 & \mathrm{~F} & 37\end{array}$

5.6

13 Anaplastic astrocytoma

$3 \quad$ M 52

1.1

14* Oligodendroglioma

$\begin{array}{lll}2 & \mathrm{~F} & 43\end{array}$

6.1

Note. Pathological data were not available for Participant 5. * indicates the participant with right-hemisphere dominance for language. NA** = not a tumour. 
Table 2. Amount of damage to different frontal and temporal grey- and white-matter regions, expressed as a percentage. $\mathrm{ACC}=$ anterior cingulate; $\mathrm{IFG}=$ inferior frontal gyrus; oper $=$ pars opercularis; orb $=$ pars orbitalis; tri $=$ pars triangularis; $\mathrm{MFG}=$ middle frontal gyrus; $\mathrm{SFG}=$ superior frontal gyrus; ITG = inferior temporal gyrus; $\mathrm{MT}(\mathrm{G})=$ middle temporal (gyrus); $\mathrm{ST}(\mathrm{G})=$ superior temporal (gyrus); $\mathrm{AF}=$ arcuate fasciculus; FOF $=$ frontal-occipital fasciculus; ILF = inferior longitudinal fasciculus

\begin{tabular}{|c|c|c|c|c|c|c|c|}
\hline $\begin{array}{l}\text { Particip } \\
\text { ant }\end{array}$ & $\mathrm{ACC}$ & $\begin{array}{l}\text { IFG } \\
\text { oper }\end{array}$ & IFG orb & IFG tri & MFG & SFG & Insula \\
\hline 1 & 0 & 0 & 0 & 0 & 0 & 0 & 0 \\
\hline 2 & 0 & 10.5 & 6.3 & 3.8 & 0 & 0 & 78.6 \\
\hline 3 & 0 & 0 & 0 & 0 & 0 & 0 & 0 \\
\hline 4 & 5.9 & 2.7 & 45.9 & 1.6 & 0 & 0 & 56.6 \\
\hline 5 & 34.5 & 0 & 0 & 0 & 11.1 & 35.7 & 0 \\
\hline 6 & 0 & 0.7 & 0 & 0 & 0 & 0 & 0.9 \\
\hline 7 & 0 & 4.8 & 0 & 15.2 & 20.2 & 2.6 & 0 \\
\hline 8 & 0 & 0 & 0 & 0 & 0 & 0 & 0 \\
\hline 9 & 0 & 0 & 13.8 & 0 & 0 & 0 & 14.8 \\
\hline 10 & 0 & 8.2 & 0 & 27.9 & 3.7 & 0 & 0 \\
\hline 11 & 0 & 0 & 0 & 0 & 17.8 & 14.6 & 0 \\
\hline 12 & 0 & 0.3 & 13.8 & 0.3 & 0 & 0 & 70 \\
\hline 13 & 0 & 0 & 0.4 & 0.1 & 0 & 0 & 15.3 \\
\hline 14 & 0 & 72.3 & 9.2 & 47.5 & 0.7 & 0 & 84.3 \\
\hline $\begin{array}{l}\text { Particip } \\
\text { ant }\end{array}$ & ITG & \multicolumn{2}{|l|}{ MTG } & MT pole & STG & \multicolumn{2}{|r|}{ ST pole } \\
\hline 1 & 44.3 & \multicolumn{2}{|l|}{1.2} & 0 & 0 & \multicolumn{2}{|r|}{0} \\
\hline 2 & 0 & \multicolumn{2}{|l|}{0.6} & 20.6 & 14.1 & \multicolumn{2}{|r|}{61.1} \\
\hline 3 & 41.1 & \multicolumn{2}{|l|}{14.6} & 0 & 0 & \multicolumn{2}{|r|}{0} \\
\hline 4 & 0 & \multicolumn{2}{|l|}{0} & 0 & 3.8 & \multicolumn{2}{|r|}{29.8} \\
\hline 5 & 0 & \multicolumn{2}{|l|}{0} & 0 & 0 & \multicolumn{2}{|r|}{0} \\
\hline 6 & 0 & \multicolumn{2}{|l|}{0.2} & 0 & 20.9 & \multicolumn{2}{|r|}{0} \\
\hline 7 & 0 & \multicolumn{2}{|l|}{0} & 0 & 0 & \multicolumn{2}{|r|}{0} \\
\hline 8 & 0.2 & \multicolumn{2}{|l|}{0} & 0 & 0 & \multicolumn{2}{|r|}{0} \\
\hline 9 & 0 & \multicolumn{2}{|l|}{0.2} & 20.1 & 0 & & 23.5 \\
\hline 10 & 0 & 0 & & 0 & 0 & & 0 \\
\hline 11 & 0 & 0 & & 0 & 0 & & 0 \\
\hline 12 & 0 & 0.7 & & 35.3 & 13.4 & & 40.4 \\
\hline 13 & 6.3 & 43.9 & ( & 0 & 55.7 & & 19.6 \\
\hline 14 & 0 & 0.1 & & 14.7 & 8.1 & & 47.4 \\
\hline $\begin{array}{l}\text { Particip } \\
\text { ant }\end{array}$ & $\begin{array}{l}\text { AF Long } \\
\text { segment }\end{array}$ & & $\begin{array}{l}\text { F Posterio } \\
\text { gment }\end{array}$ & $\mathrm{FC}$ & & ILl & \\
\hline 1 & 0.7 & 0. & & 0. & & 5.2 & \\
\hline 2 & 0.9 & 0 . & & 2. & & 3.7 & \\
\hline
\end{tabular}




\begin{tabular}{lllll}
3 & 6.5 & 3.7 & 0.1 & 3.1 \\
4 & 0.1 & 0.0 & 10.2 & 0.6 \\
5 & 0.0 & 0.0 & 0.7 & 0.0 \\
6 & 2.0 & 1.7 & 0.0 & 0.0 \\
7 & 0.0 & 0.0 & 0.0 & 0.0 \\
8 & 0.0 & 0.0 & 1.9 & 12.9 \\
9 & 0.0 & 0.0 & 2.4 & 2.7 \\
10 & 2.2 & 0.0 & 0.0 & 0.0 \\
11 & 0.0 & 0.0 & 0.0 & 0.0 \\
12 & 0.0 & 0.0 & 4.8 & 5.4 \\
13 & 21.7 & 23.2 & 3.2 & 4.5 \\
14 & 3.4 & 0.0 & 9.4 & 4.9 \\
\hline
\end{tabular}

